\title{
Sevoflurane-induced changes in infants' quantifiable electroencephalogram \\ parameters
}

\section{Running head (short title)}

Pediatric aEEG and sevoflurane concentration

Article category

Original research

\section{Authors}

Stephen McKeever ${ }^{123}$

Linda Johnston ${ }^{234}$

Andrew J. Davidson ${ }^{12}$

\section{Affiliations}

${ }^{1}$ Department of Anaesthesia and Pain Management, Royal Children's Hospital, Melbourne, Australia

${ }^{2}$ Murdoch Childrens Research Institute, Melbourne, Australia

${ }^{3}$ Melbourne School of Health Sciences, The University of Melbourne, Australia

${ }^{4}$ School of Nursing \& Midwifery, Queen's University Belfast, United Kingdom

Correspondence

Dr. S. McKeever

Department of Anaesthesia and Pain Management

Royal Children's Hospital

50 Flemington Road

Parkville

3052

Victoria

Australia

stephen.mckeever@rch.org.au 


\section{Abstract \\ Background}

Electroencephalogram (EEG) based depth of anesthesia algorithms developed in the adult population have not demonstrated the same reliability when applied to infants. This may be due to frequency changes occurring in the EEG during development. Amplitude-integrated EEG (aEEG) is based primarily in the time domain and hence may have greater utility in infants.

\section{Objective}

To investigate the relationship between age adjusted Minimal Alveolar Concentration (MAC) multiples and $\mathrm{aEEG}$ in children under two years of age

\section{Methods}

The aEEG, Spectral Edge Frequency 90\% (SEF90) and Bispectral Index ${ }^{\mathrm{TM}}$ (BIS) were investigated in a prospective study of children less than two years of age. After anesthetic induction, and caudal block administration, EEG data were collected simultaneously with BrainZ BRM2 ${ }^{\mathrm{TM}}$ and $\mathrm{BIS} \mathrm{S}^{\mathrm{TM}}$ monitors. Using a randomized crossover design, children received up to three age adjusted concentrations of sevoflurane: $0.75,1$ and 1.25 MAC. After 15 minutes of stable anesthetic delivery EEG readings were obtained. Prediction Probability $\left(\mathrm{P}_{\mathrm{k}}\right)$ and correlation coefficients were calculated for each EEG parameter.

Results

From 51 children 102 stable anesthetics concentrations were obtained. For all age groups $\mathrm{Pk}$ of aEEG to multiple of age adjusted MAC was less than 0.72 indicating a poor predictive power for aEEG. In contrast for the SEF90 and BIS there was evidence for better predictive properties in children aged between 6 months and 2 years, with a $P_{k}$ greater than 0.81 .

\section{Conclusion}

The aEEG is unlikely to be a useful measure of anesthesia depth in young children.

\section{MeSH-compliant keywords}

Electroencephalography, inhalation, anesthesia, consciousness, intraoperative, monitoring 


\section{Background}

For older children, electroencephalogram (EEG) depth of anesthesia monitors usually have performance characteristics similar to adults(1), whilst in children less than two years of age these monitors have poorer performance(2). This may be because the EEG changes with increasing age(3, 4). Many anesthetic depth monitors interpret information from EEG frequency and time domains $(5,6)$, whilst others evaluate the EEG's relative entropy (7). Due to the maturational changes in dominant frequency it is plausible that methods that do not rely on frequency domains may be more appropriate in younger children.

The Cerebral Function Monitor (8), and similar monitors such as the BrainZ BRM (Natus Medical Incorporated, San Carlos, CA, USA), examine EEG primarily in the time domain where the primary output parameter is the amplitude-integrated EEG (aEEG). Obtained from rectified raw EEG the aEEG reflects peak-to-peak EEG fluctuations and is obtained using an envelope detection algorithm (8). Increasingly used in neonatal units, these monitors are used for seizure detection and brain function monitoring(9). These monitors predominately reflect EEG information in the time domain and therefore may provide a useful method of EEG interpretation for children during anesthesia.

Two studies have examined aEEG during pediatric anesthesia $(10,11)$. These observational studies found, that in a heterogeneous population of children undergoing a variety of anesthetics, aEEG could roughly discriminate between awake and anesthetized states. However, these studies were not designed to test other aspects of depth measure such as the aEEG's ability to differentiate between different anesthetic concentrations.

This study aimed to investigate relationships between age adjusted Minimal Alveolar Concentration (MAC) multiples and aEEG in children under two years of age. 


\section{Methods}

Patients

This study was approved by Human Research Ethics Committee at The Royal Children's Hospital, Melbourne, reference number 29109 , on $30^{\text {th }}$ September 2009. Informed, written consent was obtained from the child's parent. Eligible children were aged less than two years of age, American Society of Anesthesiologists Physical Status Classification System I or II, scheduled for surgery or a procedure under general anesthesia which was expected to last at least 20 minutes where the planned anesthetic involved no premedication other than acetaminophen, induction with sevoflurane \pm nitrous oxide, a caudal local anesthetic block without clonidine and sevoflurane alone for maintenance anesthesia. Other study exclusion criteria were: known neurological disorder, born earlier than 36 weeks gestational age, receiving any sedative medication, receiving beta blockers, or a contraindication to receiving sevoflurane such as a family history of malignant hyperthermia.

Owing to the exploratory nature of this study no a-priori power calculation was undertaken as the likely differences in EEG values were unknown. Based on previous studies performed at our institution we estimated that we could recruit 72 children in the period when we had staff for the project. There were to be 18 children recruited in each of four age groups: one day to 31 days $(<1$ month), 32 days to 182 days (1-6 months), 183 days to 365 days (6-12 months), 366 days to 730 days (1-2 years).

\section{Data acquisition and electrophysiological monitoring}

EEG sensors were applied post induction in the $F_{p 1}-F_{p 2}$ (forehead) and either the $C_{3}-P_{3}$ (left) or $C_{4}-P_{4}$ (right) regions according to the international 10-20 system, with a reference electrode behind the ear. BrainZ ${ }^{\text {TM }}$ Hydrogel Sensors (Natus Medical Incorporated, San Carlos, CA, USA) were applied after sensor site exfoliation and an application of conductive paste. Sensors were applied while the child was lying on their side for the caudal local anesthetic block. A four electrode BIS sensor was also placed above forehead BrainZ sensors after cleansing with 70\% isopropyl alcohol.

EEG recordings were obtained using the BRM2 ${ }^{\mathrm{TM}}$ (Natus Medical Incorporated, San Carlos, CA). The $\mathrm{BRM}^{\mathrm{TM}}$ calculates SEF90 and aEEG from four second epochs obtained from the $2 \mathrm{to} 20 \mathrm{~Hz}$ range. The BIS ${ }^{\mathrm{TM}}$ monitor used was the A-2000 XP Platform, software version 3.1, (Covidien, Boulder, CO, 
USA) using the standard four sensors. BIS smoothing rate was set at 15 seconds. Infant's physiological parameters, and gas concentration data, including end-tidal sevoflurane values were obtained at 30 second intervals and transferred directly from the Aisys ${ }^{\circledR}$ anesthetic machine (GE Healthcare, Finland) to a laptop using S/5 collect software version 4 (GE Healthcare, Finland). Anesthesia protocol

Induction of anesthesia was with sevoflurane \pm nitrous oxide. Nitrous oxide was discontinued after placement of a tracheal tube or laryngeal mask. At an appropriate time (i.e. when the child was stable from a cardiovascular and respiratory perspective, and the anesthetist was satisfied that the caudal anesthetic was working), the anesthetist then aimed for a proscribed end-tidal sevoflurane concentration. The child's scheduled operation and other procedures continued as per usual practice during data collection. Prior to study commencement target end-tidal sevoflurane concentrations were generated using stratified randomization and sealed in sequentially numbered coded opaque envelopes.

Randomization generated three different end-tidal concentrations to be aimed for as the duration of the operation permitted. It was not expected that all participants would achieve all three sevoflurane end-tidal concentrations, hence randomization aimed to ensure an approximately even distribution of end-tidal concentrations across age groups.

Children's MAC changes with age and younger children require more sevoflurane. Thus, anesthetic concentrations represented roughly similar levels of potency across ages. Target end-tidal concentrations were calculated as multiples of the age adjusted MAC $(0.75,1$ and $1.25 \mathrm{MAC})$. MAC multiples were those recommended by Abbott Laboratories(12) and listed in Table 1. Awakening occurs at about $1 / 3$ MAC and thus as these children were to have their surgical stimulus ablated with a caudal block they were not expected to move or awaken when given 0.75 MAC. End-tidal sevoflurane concentrations were held stable for 15 minutes to allow equilibrium between the end-tidal and effect site concentrations. In addition, this stable period accounted for potential delays inherent in signal processing. After 15 minutes of stable end-tidal sevoflurane, monitors and laptop were event marked accordingly. If procedure time permitted, a second and then a third randomized concentration were similarly targeted. 
Attending anesthetists were blinded to EEG information as monitors were turned away from their line of sight. Decisions about anesthetic management were made as per standard practice. Anesthetists, using usual clinical assessment, could override the protocol if they judged that the child had inadequate or excessive anesthetic depth.

Data analysis

Offline, individual patient BRM2 ${ }^{\mathrm{TM}}$ files were reviewed using AnalyzeResearch 1.7 (BrainZ Instruments Limited; Auckland, New Zealand). SEF90, aEEGmean, Mains Hum, and Impedance data in averaged 1 minute epochs, along with event markings, were exported to Microsoft巴 Excel 2007. BIS data in 1 minute epochs, as an average of the preceding minute, along with event marks were retrieved using WinHist@ software Version 2.01 (Covidien, Boulder, CO, USA). For individual patients an Excel file was created that collated event marks, BRM2, BIS and anesthetic machine data. From these files EEG and anesthetic data points were established that occurred at relevant event marks and complied in Microsoft $\circledast$ Access 2007 before being imported into Matlab® Version 7.7.0 (R2008b) for analysis.

A bespoke Matlab program filtered and removed BRM2 ${ }^{\mathrm{TM}}$ data with periods of Impedance $>10 \mathrm{k} \Omega$, or excessive electrical interference in the $50 \mathrm{~Hz}$ range (Mains Hum $>50 \mu \mathrm{Vpp}$ ). Also removed were BIS data with a signal quality index $<50$ or electromyography $>50 \mathrm{~dB}$.

\section{Statistical analysis}

Considering age adjusted multiples of MAC as dependent variables EEG parameter performance as a potential anesthetic depth indicator was summarized using the Prediction Probability $\left(P_{K}\right)$ statistic $(13)$. EEG parameters completely concordant with multiple of MAC then the $P_{k}$ would be 1 . Conversely, if an EEG parameter is completely discordant with age adjusted multiple of MAC then the $P_{k}$ would be 0. If the probability of discordance equals the concordance, the $P_{k}$ will be 0.5 ; i.e. the indicator value only correctly predicts the dependent variable $50 \%$ of the time. A $P_{K}$ of 0.5 is equivalent to flipping a coin. Therefore, $P_{k}$ values further from 0.5 are of particular interest. $P_{k}$ was computed with PKMACRO using Microsoft Office Excel® 2007. Where appropriate $P_{k}$ values were then transformed using 1$P_{k}(14) . P_{k}$ standard errors and hence $95 \%$ confidence intervals were obtained using the Jackknife method (13). Strength of correlation between age adjusted multiple of MAC and EEG parameters was 
also determined using the Spearman correlation coefficient. Spearman correlation coefficients and $P_{k}$ values were reported in each age group for aEEGmean, SEF90 and BIS. 


\section{Results}

Between October 2009 and September 2010, 69 families were approached to participate in the study and consent was obtained from 55 (79.7\%) families. During the recruitment period, a multi-center international collaborative study; with inclusion criterion similar to this study, also aimed to enroll children aged less than 31 days. The study protocols did not permit families to be in both studies and priority was given to the multi-center study. Thus, only one child was enrolled in this group giving insufficient data to evaluate children aged less than 31 days. This child was not included in subsequent analysis. One child received clonidine in their caudal and was excluded from the analysis. Two other children were excluded due to an ineffective caudal block. There were no circumstances where the anesthetist abandoned the target concentration protocol due to clinically inappropriate depth of anesthesia. Details of the 51 children included in the analysis can be found in Table 2 . There were a higher proportion of males than females. Younger children were more likely to receive inguinal hernia repairs and older children received more hypospadias, chordee or orchidopexy repairs. Filtering EEG data further minimized samples available for analysis, as shown in Table 3. In the 10 minutes prior to EEG sampling median end tidal sevoflurane concentrations were either at or within $0.1 \%$ (IQR $0.1 \%$ ) of the target concentration. The aEEGmean, SEF90 and BIS values are shown in figure 1.

\section{Assessment of EEG parameters prediction probability of multiple of MAC}

The aEEGmean generally demonstrated low prediction probability across all ages (Table 4). $P_{k}$ of SEF90 and BIS was also low in children aged one to six months. In the two older age groups BIS and SEF90 had better prediction probability than aEEGmean. Parietal montage performance was not substantially different to frontal montage.

\section{Correlation between multiple of MAC and EEG parameters}

There was no significant correlation between aEEGmean and age adjusted multiple of MAC in any age group (Table 4). Similarly there was no correlation between SEF90 or BIS and age adjusted multiple of MAC in children aged one to six months. In the two older age groups there was a weak correlation between both BIS and SEF90 and multiple of MAC. Once again performance in the parietal montage performance was not substantially different to frontal montage. 


\section{Discussion}

This study found that in children less than two years of age the aEEGmean was not correlated with or able to predict age adjusted multiple of MAC. This would imply that alone aEEGmean is unlikely to be a useful indicator of anesthesia depth in this age group. In contrast, SEF90 and BIS did show some weak correlation with and ability to predict age adjusted multiple of MAC in children aged between six months and two years.

The concept of anesthesia depth is controversial and anesthesia depth does not represent any discrete physiological phenomenon. As such there is no gold standard for anesthesia depth, and assessing monitors or EEG parameters as measures of depth is inherently problematic. One method to assess the performance of anesthesia depth indices is to determine the prediction coefficient or simple correlation between parameter and a known variable anesthetic concentration in a setting where there was no or minimal surgical stimulation(15). In this study we used specified end tidal sevoflurane concentrations allowing 15 minutes for equilibration between end tidal and effect site concentrations. Greater anesthesia concentration should result in a monotonic change in index. Ideally anesthesia depth indices should also reflect clinically relevant measures of hypnosis during arousal; this is particularly relevant if such an index is to be used to guide sedation or to determine if the child is conscious or not. Unfortunately, such clinical endpoints are very difficult to define in small children. It might be argued that indices just need to predict whether or not the patient is conscious or unconscious. Our previous studies demonstrated that the aEEG may indeed be able to do this $(10$, 11). Such a simple approach however ignores the potential to assess the underlying degree of depth or "rousability" in the unconscious patient. A "deep" subject is unlikely to become conscious even with greater surgical stimulus while a "light" subject may arouse and become conscious. Similarly an "excessively deep" subject may have a concentration of anesthetic greater than needed to prevent consciousness even with the strongest of stimuli. Once again, it is controversial whether or not this degree of "rousability" actually exists or reflects any real physiological process. Thus a change in index may simply be measuring an epiphenomenon or direct effect of the drug on the EEG.

Regardless of the mechanistic uncertainty it is still clinically worthwhile to explore the degree to which an index changes with drug concentration in an unconscious subject. 
As mentioned above, a previous exploratory study(11) investigated the aEEG in a heterogeneous population of children during anesthesia and found that, for children less than two years of age, aEEG was able to discriminate between awake and anaesthetized states. This previous exploratory study did not permit a robust examination of aEEG or SEF90 according to anesthetic concentration. Thus, this current study was conducted. It appears that while aEEG is able to differentiate between the gross states of awake and anesthetized, aEEG is not sensitive changes occurring between MAC 0.75 and MAC 1.25. While this could imply aEEG is unable to predict depth of anesthesia in an unconscious child, it is also possible that at this level the dose response curve is fairly flat and thus does not rule out a possible ability to predict doses across a wider or different range of concentrations(16). We chose MAC 0.75 to MAC 1.25 as these are the concentrations around which most children would be anesthetized and hence are the most clinically relevant. Detecting changes at several multiples of MAC is clinically less relevant. It is possible that the aEEG could detect differences between much lower or higher concentrations however these studies are logistically and ethically challenging as children may awake or develop larygospasm at lower concentrations or have significant hypotension at high concentrations. If the aEEG does not perform well around MAC then performing these more difficult studies cannot be justified.

Several other studies have examined the $\mathrm{P}_{\mathrm{k}}$ of BIS or spectral edge frequency during anesthesia, however when comparing studies it is important to note that $\mathrm{P}_{\mathrm{k}}$ values are dependent on the granularity of the scale used and hence it is problematic to compare $P_{k}$ values between studies where different points of anesthesia depth are measured. $P_{k}$ values in our study can be compared within the study, but should be cautiously compared to values obtained in other studies. $P_{K}$ of BIS to sevoflurane has been studied during adult anesthesia, but there are fewer studies in the pediatric population. During adult anesthesia, Ellerkmann et al. (17) and Soehle et al. (18) both found that BIS, within the sevoflurane range zero to $5 \%$, had a $\mathrm{P}_{\mathrm{k}}$, of 0.8 . During pediatric anesthesia, BIS can demonstrate an acceptable $P_{k}$ between the states of awake or emergence and being anaesthetized with sevoflurane(19) similar to that found in adult studies(15). This current study found that $P_{k}$ of BIS was lesser than in previous adult studies and particularly small in the one to six month age group. This confirms the previous evidence regarding BIS's poor performance in younger children. Few studies have assessed the $P_{k}$ of spectral edge frequency during inhaled anesthesia, and none during 
pediatric anesthesia. During adult anesthesia with sevoflurane, Katoh and colleagues(15) reported that the $\mathrm{P}_{\mathrm{k}}$ of SEF95 was 0.81 , though the Pk of movement after skin incision was only 0.57 . As mentioned above; comparing $\mathrm{P}_{\mathrm{k}}$ between studies is of questionable validity.

Previous studies in the pediatric population have explored the relationship between end-tidal sevoflurane and the BIS(1, 20-24). Many of these studies have shown that with increasing sevoflurane concentration there is a corresponding fall in BIS values $(1,20,21)$. However, interindividual and age related differences have also been observed $(1,21,22)$. At the end of surgery, Davidson and colleagues(23) administered reducing doses of sevoflurane to infants (five to 11 months) and children (12 - 178 months). BIS values of children related to reducing anesthetic concentration whereas in infants they did not. Consistent with previous findings, our study found that BIS above six months was moderately correlated to increasing steady state sevoflurane concentration. However, BIS of children aged one to six months did not change with increasing endtidal sevoflurane. It is unclear why BIS performs poorly in infants. This may be related to the changing function of receptors in this age group and changing maturity of connectivity; for example there are changes in GABA function(25) and associated $\mathrm{GABA}_{\mathrm{A}}$ receptors (26).

Similar to previous studies, this study has shown an age-related change in BIS $(24,27)$. However, in contrast to previous findings, this study reports a positive correlation of age to BIS, obtained whilst anaesthetized, in children up to two years of age. Kim and colleagues(24) reported a reduction in BIS with increasing age in children, between six months and 12 years of age, receiving sevoflurane doses that were not age adjusted. As sevoflurane requirement reduces with age, it is possible that the endtidal sevoflurane concentrations of 2.0, 3.0 and 4.0\%, delivered by Kim and colleagues, may have achieved deeper levels of anesthesia with increasing age. Therefore, as age increased, lower BIS values would have been generated. Tirel and colleagues(27) examined the correlation of BIS values with increasing age and sevoflurane MAC multiples. This study of children six months to 14 years, also demonstrated a reduction in BIS values with increasing age. The conflicting results of the study by Tirel and colleagues and our study could be related to the age range of participants. Tirel and colleagues investigated children six months to 14 years whilst our study examined a younger 
population of children 22 days to two years of age. This could indicate the presence of an initial rise then a fall of BIS values with increasing age that peaks around two years of age.

This study has several limitations. Processing within the BRM2 ${ }^{\mathrm{TM}}$ involves $\mathrm{EEG}$ information being passed through an asymmetrical filter. This filter strongly attenuates activity below $2 \mathrm{~Hz}$ and above 20 $\mathrm{Hz}$ (8). Filtering less than $2 \mathrm{~Hz}$ was originally introduced to remove low frequency artifact caused by sweating (8). The above $20 \mathrm{~Hz}$ filter was intended to remove artifact such as muscle activity (8). Some EEG activity relevant to monitoring of EEG during anesthesia may occur outside of these ranges. However, examination of the EEG within the $2-20 \mathrm{~Hz}$ range reduces artifact and reduces the signal-to-noise ratio with associated improvements in analysis reliability(28). Future studies are indicated to investigate the clinical relevance of EEG information outside the $2-20 \mathrm{~Hz}$ range. However, from a pragmatic point of view these aEEG monitors are increasingly being used in the neonatal units and we wished to assess a parameter that could be quickly extended to intra-operative use.

As mentioned above there is no 'gold standard' with which to assess children's depth of anesthesia (29). Sevoflurane MAC multiples were chosen as a guide within the realms of usual anesthetic practice and age adjusted according to manufacturers recommendations (12). Age adjusted MAC values are based on relatively limited data and thus it is unclear how accurate they are. We chose the manufacturer's recommended age related MAC values as we assumed most would follow these values. As the main aim is to generate $P_{k}$ within each age group, and not to compare aEEG at specific concentrations across age groups, then inaccuracies in age related MAC adjustments should not substantially impact the validity of the study. A further limitation is the possibility that the end-tidal sevoflurane values did not reflect brain concentration, especially if there is a leak with high gas flows or in the presence of high respiration rates.

Lastly, this study only looked at one simple measure of aEEG. It is possible that other aspects of aEEG or other aspects of the time domain may still be of value. Similarly, it is possible that elements of the aEEG may be useful in forming composite scales that use multiple EEG elements. One EEG element worthy of further investigation is burst suppression. The presence of burst suppression will 
reduce aEEG mean, and also widen the difference between aEEG minimum and maximum. Thus the aEEG may be useful in detecting burst suppression. The detection of burst suppression is however difficult in small children as the overall amplitude is less and some children develop a discontinuous EEG that mimics burst suppression (9).

In conclusion, this study found the aEEGmean was not a predictor of sevoflurane concentration in children less than two years of age. This suggests that this aspect of aEEG is not a useful candidate measure of anesthesia depth for infants.

\section{Acknowledgements}

This study was approved by Human Research Ethics Committee at The Royal Children's Hospital, Melbourne, reference number 29109, on 30 ${ }^{\text {th }}$ September 2009.

The project was supported by the Victorian Government's Operational Infrastructure Support Program. Dr. McKeever received Postgraduate Health Research Scholarships from the Murdoch Childrens Research Institute, Melbourne and a Trust Fund Faculty Research Scholarship from the Faculty of Medicine, Dentistry and Health Sciences at The University of Melbourne

Authors have no conflict of interest related to this paper.

Table 1 Target concentrations of end-tidal sevoflurane

\begin{tabular}{ccc}
\hline Age group & $\begin{array}{c}\text { Multiples of } \\
\text { Minimum Alveolar } \\
\text { Concentration }\end{array}$ & $\begin{array}{c}\text { Target end-tidal } \\
\text { sevoflurane } \\
\text { concentration }\end{array}$ \\
\hline One to six month/s & $0.75 \mathrm{MAC}$ & $2.2 . \%$ \\
& $1 \mathrm{MAC}$ & $3.0 \%$ \\
\hline Six months to one year & $1.25 \mathrm{MAC}$ & $3.7 \%$ \\
\hline One to two year/s & $0.75 \mathrm{MAC}$ & $2.1 \%$ \\
& $1 \mathrm{MAC}$ & $2.8 \%$ \\
& $1.25 \mathrm{MAC}$ & $3.5 \%$ \\
\hline & $0.75 \mathrm{MAC}$ & $1.9 \%$ \\
& $1 \mathrm{MAC}$ & $2.6 \%$ \\
& $1.25 \mathrm{MAC}$ & $3.2 \%$ \\
\hline
\end{tabular}


Table 2 Infant data of children included in the analysis

\begin{tabular}{lccc}
\hline & $\begin{array}{c}\text { One to six } \\
\text { month/s }\end{array}$ & $\begin{array}{c}\text { Six months } \\
\text { to one year }\end{array}$ & $\begin{array}{c}\text { One to } \\
\text { two year/s }\end{array}$ \\
\hline Total recruited in each group & 18 & 17 & 16 \\
Age in days, mean (SD) & $83(25.8)$ & $259(39.7)$ & $490(67.2)$ \\
Male & 16 & 16 & 16 \\
ETT/LMA & $14 / 4$ & $13 / 4$ & $6 / 10$ \\
Procedure & & & \\
Inguinal hernia repair & 17 & 2 & 1 \\
Hypospadias / chordee repair & - & 13 & 12 \\
Hydrocele repair & - & - & 1 \\
Circumcision with cystoscopy & 1 & - & - \\
Orchidopexy & - & 2 & 2 \\
& & & \\
\hline
\end{tabular}

LMA: Laryngeal Mask Airway, ETT: Endotracheal Tube

Table 3 EEG samples pre- and post- filtering

\begin{tabular}{lccc}
\hline & $\begin{array}{c}\text { One to six } \\
\text { month/s }\end{array}$ & $\begin{array}{c}\text { Six months } \\
\text { to one year }\end{array}$ & $\begin{array}{c}\text { One to } \\
\text { two year/s }\end{array}$ \\
\hline $\begin{array}{l}\text { Number of pre filtering stable sevoflurane } \\
\text { EEG samples obtained }\end{array}$ & 23 & 38 & 41 \\
Number of post filtering EEG & & & \\
values analyzed & & & \\
BRM2 parietal & 20 & 38 & 32 \\
BRM2 forehead & 23 & 38 & 39 \\
BIS & 23 & 38 & 41 \\
\hline
\end{tabular}

Table 4 Spearman correlation coefficients $\left(r^{2}\right)$ and prediction probability $\left(P_{k}\right)$ for MAC multiples and EEG parameters according to age group

\begin{tabular}{|c|c|c|c|c|c|c|c|c|c|}
\hline \multirow{3}{*}{ Age group } & \multirow{3}{*}{$\begin{array}{c}\text { EEG } \\
\text { Parameter }\end{array}$} & \multicolumn{4}{|c|}{ Forehead montage } & \multicolumn{4}{|c|}{ Parietal montage } \\
\hline & & \multicolumn{2}{|c|}{$\begin{array}{l}\text { Spearman } \\
\text { correlation }\end{array}$} & \multicolumn{2}{|c|}{$\begin{array}{l}\text { Prediction } \\
\text { probability }\end{array}$} & \multicolumn{2}{|c|}{$\begin{array}{l}\text { Spearman } \\
\text { correlation }\end{array}$} & \multicolumn{2}{|c|}{$\begin{array}{l}\text { Prediction } \\
\text { probability }\end{array}$} \\
\hline & & $r^{2}$ & $P$ value & $P_{k}$ & $95 \% \mathrm{Cl}$ & 2 & $P$ value & $P_{k}$ & $95 \% \mathrm{Cl}$ \\
\hline \multirow{3}{*}{$\begin{array}{l}\text { One to six } \\
\text { month/s }\end{array}$} & aEEGmean & 0.01 & 0.61 & 0.55 & $0.33,0.78$ & 0.05 & 0.35 & 0.60 & $0.35,0.85$ \\
\hline & SEF90 & 0.08 & 0.20 & 0.37 & $0.15,0.59$ & 0.01 & 0.69 & 0.52 & $0.33,0.71$ \\
\hline & BIS & 0.01 & 0.59 & 0.56 & $0.32,0.80$ & & & & \\
\hline \multirow{3}{*}{$\begin{array}{l}\text { Six months } \\
\text { to one year }\end{array}$} & aEEGmean & 0.10 & 0.05 & 0.65 & $0.50,0.80$ & 0.18 & 0.007 & 0.71 & $0.56,0.85$ \\
\hline & SEF90 & 0.64 & $<0.0001$ & 0.91 & $0.84,0.98$ & 0.78 & $<0.0001$ & 0.96 & $0.92,0.99$ \\
\hline & BIS & 0.43 & $<0.0001$ & 0.82 & $0.72,0.91$ & & & & \\
\hline \multirow{3}{*}{$\begin{array}{c}\text { One to } \\
\text { two year/s }\end{array}$} & aEEGmean & 0.02 & 0.39 & 0.58 & $0.41,0.74$ & 0.10 & 0.085 & 0.56 & $0.40,0.71$ \\
\hline & SEF90 & 0.34 & 0.0001 & 0.75 & $0.62,0.89$ & 0.29 & 0.002 & 0.67 & $0.53,0.82$ \\
\hline & BIS & 0.24 & 0.0012 & 0.71 & $0.60,0.82$ & & & & \\
\hline
\end{tabular}



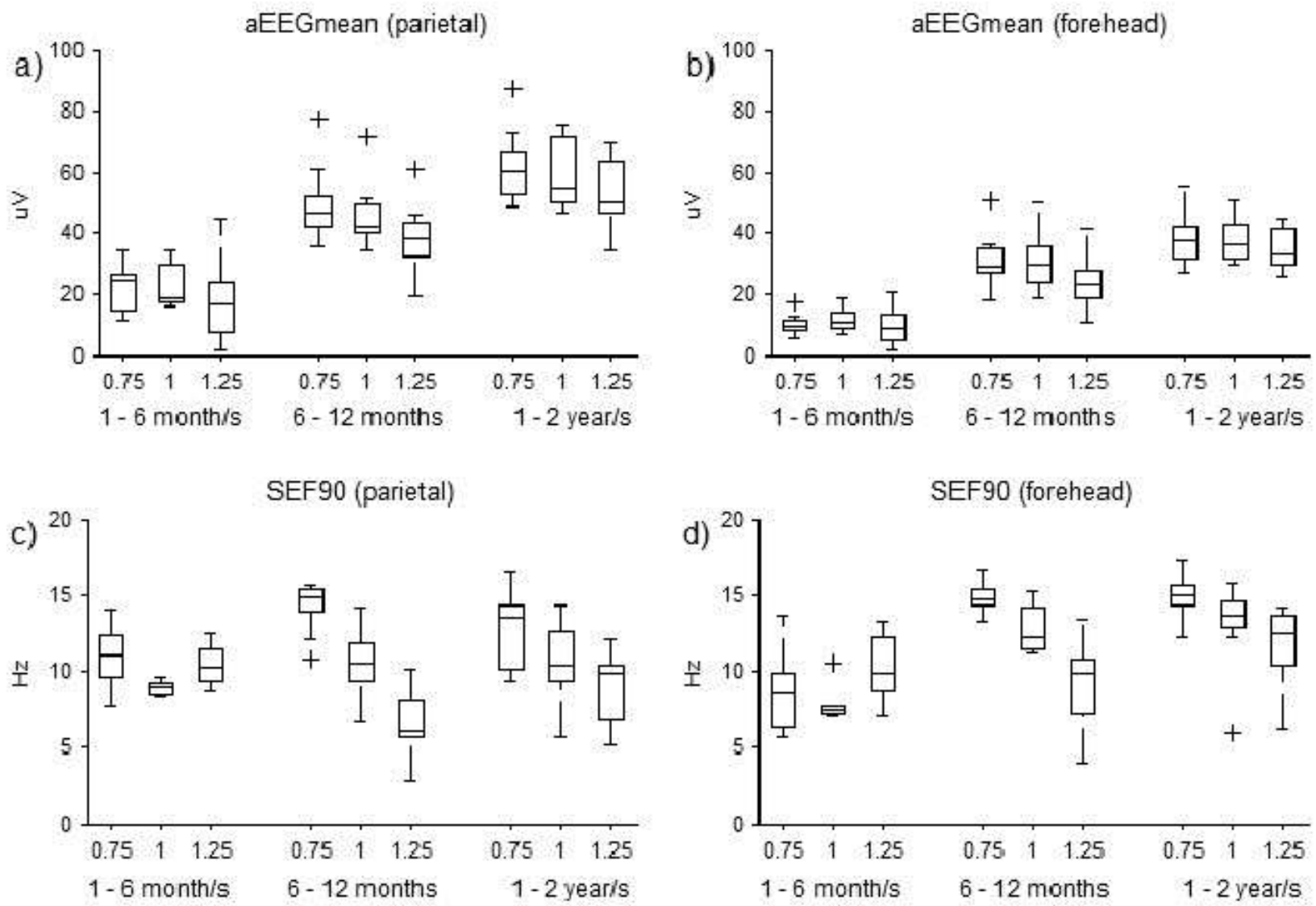

BIS

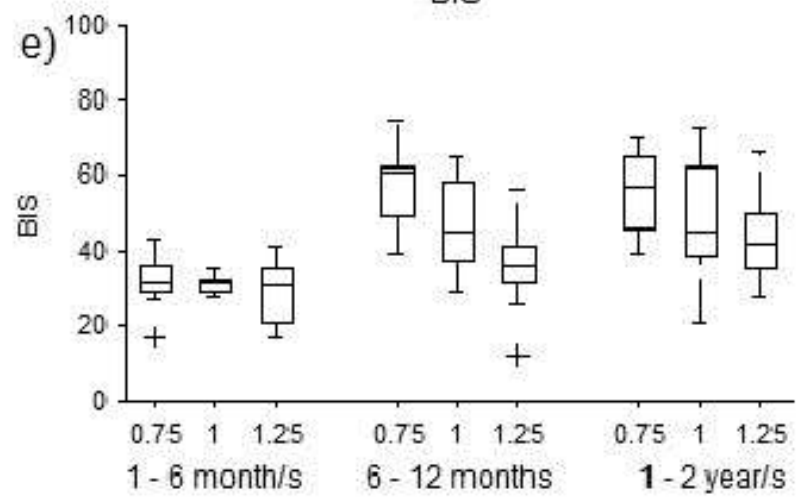

Figure 1 Figure 1 Box (25th and 75th percentile), whisker plots (extreme data points not considered outliers and outliers (+) of aEEGmean, SEF90 and BIS values compared to multiples of MAC of individual age groups (parietal and forehead). 


\section{References}

1. Denman WT, Swanson EL, Rosow D, Ezbicki K, Connors PD, Rosow CE. Pediatric evaluation of the Bispectral Index (BIS) monitor and correlation of BIS with end-tidal sevoflurane concentration in infants and children. Anesthesia \& Analgesia. 2000;90(4):872-7.

2. Davidson AJ. Monitoring the anaesthetic depth in children - an update. Current Opinion in Anaesthesiology. 2007;20(3):236-43.

3. Bell MA. The ontogeny of the EEG during infancy and childhood: Implications for cognitive development. In: Garreau B, editor. Neuroimaging in child neuropsychiatric disorders. Berlin: Springer-Verlag; 1998. p. 97-111.

4. Matoušek M, Petersén I. Frequency analysis of the EEG in normal children and in normal adolescents. In: Kellaway P, Petersén I, editors. Automation of Clinical Electroencephalography. New York: Raven Press; 1973. p. 75-102.

5. Rampil IJ. A primer for EEG signal processing in anesthesia. Anesthesiology. 1998;89(4):9801002.

6. Kreuer S, Wilhelm W. The Narcotrend monitor. Best Practice \& Research: Clinical Anaesthesiology. 2006;20(1):111-9.

7. Bein B. Entropy. Best Practice \& Research: Clinical Anaesthesiology. 2006;20(1):101-9.

8. Prior P. Monitoring cerebral function: Long-term recordings of cerebral electrical activity. Amsterdam: Elsevier; 1979.

9. Hellström-Westas L, de Vries LS, Rosén I. Atlas of Amplitude-Integrated EEGs in the Newborn. 2nd ed. London: Informa Healthcare; 2006.

10. Davidson AJ, Sale SM, Wong C, McKeever S, Sheppard S, Chan Z, et al. The electroencephalograph during anesthesia and emergence in infants and children. Paediatric Anaesthesia. 2008;18(1):60-70.

11. McKeever S, Johnston L, Davidson A. An observational study exploring amplitude-integrated electroencephalogram and Spectral Edge Frequency during paediatric anaesthesia. Anaesthesia and Intensive Care. 2012;40(2):275-84.

12. Abbott Laboratories. Product monograph: Sevorane ® AF - sevoflurane. Quebec: Abbott Laboratories Limited; 2009.

13. Smith WD, Dutton RC, Smith NT. Measuring the performance of anesthetic depth indicators. Anesthesiology. 1996;84(1):38-51.

14. Jeleazcov C, Ihmsen $H$, Schmidt J, Ammon C, Schwilden $H$, Schuttler $J$, et al. Pharmacodynamic modelling of the Bispectral Index response to propofol-based anaesthesia during general surgery in children. British Journal of Anaesthesia. 2008;100(4):509-16.

15. Katoh T, Suzuki A, Ikeda K. Electroencephalographic derivatives as a tool for predicting the depth of sedation and anesthesia induced by sevoflurane. Anesthesiology. 1998;88(3):642-50

16. Ellerkmann RK, Bruhn J, Soehle M, Kehrer M, Hoeft A, Kreuer S. Maximizing prediction probability $\mathrm{PK}$ as an alternative semiparametric approach to estimate the plasma effect-site equilibration rate constant ke0. Anesthesia \& Analgesia. 2009 Nov;109(5):1470-8.

17. Ellerkmann RK, Liermann VM, Alves TM, Wenningmann I, Kreuer S, Wilhelm W, et al. Spectral entropy and Bispectral Index as measures of the electroencephalographic effects of sevoflurane. Anesthesiology. 2004;101(6):1275-82.

18. Soehle M, Ellerkmann RK, Grube M, Kuech M, Wirz S, Hoeft A, et al. Comparison between bispectral index and patient state index as measures of the electroencephalographic effects of sevoflurane. Anesthesiology. 2008 Nov;109(5):799-805.

19. Wallenborn J, Kluba K, Olthoff D. Comparative evaluation of Bispectral Index and Narcotrend Index in children below 5 years of age. Paediatric Anaesthesia. 2007;17(2):140-7.

20. McCann ME, Bacsik J, Davidson A, Auble S, Sullivan L, Laussen P. The correlation of Bispectral Index with endtidal sevoflurane concentration and haemodynamic parameters in preschoolers. Paediatric Anaesthesia. 2002 Jul 2002;12(6):519-626.

21. Fuentes R, Cortinez LI, Struys MM, Delfino A, Munoz H. The dynamic relationship between end-tidal sevoflurane concentrations, bispectral index, and cerebral state index in children. Anesthesia and Analgesia. 2008;107(5):1573-8.

22. Wodey E, Tirel O, Bansard JY, Terrier A, Chanavaz C, Harris R, et al. Impact of age on both BIS values and EEG bispectrum during anaesthesia with sevoflurane in children British Journal of Anaesthesia 2005;94(6):810-20. 
23. Davidson AJ, McCann ME, Devavaram P, Auble SA, Sullivan LJ, Gillis JM, et al. The differences in the Bispectral Index between infants and children during emergence from anesthesia after circumcision surgery. Anesthesia \& Analgesia. 2001;93(2):326-30.

24. Kim HS, Oh AY, Kim CS, Kim SD, Seo KS, Kim JH. Correlation of Bispectral Index with endtidal sevoflurane concentration and age in infants and children. British Journal of Anaesthesia. 2005;95(3):362-6.

25. Lujan R, Shigemoto R, Lopez-Bendito G. Glutamate and GABA receptor signalling in the developing brain. Neuroscience. 2005;130(3):567-80.

26. Henschel O, Gipson KE, Bordey A. GABA ${ }_{A}$ receptors, anesthetics and anticonvulsants in brain development. CNS neurological disorders drug targets. 2008 Apr;7(2):211-24.

27. Tirel O, Wodey E, Harris R, Bansard JY, Ecoffey C, Senhadji L. The impact of age on bispectral index values and EEG bispectrum during anaesthesia with desflurane and halothane in children. British Journal of Anaesthesia. 2006 April 1, 2006;96(4):480-5.

28. Inder TE, Buckland L, Williams CE, Spencer C, Gunning MI, Darlow BA, et al. Lowered electroencephalographic spectral edge frequency predicts the presence of cerebral white matter injury in premature infants. Pediatrics. 2003;111(1):27-33.

29. Davidson AJ. Measuring anesthesia in children using the EEG. Paediatric Anaesthesia. 2006;16(4):374-87. 